\title{
Performance Analysis and Comparison of Multi Hop Token Ring and Token Bus LAN Technologies
}

\author{
Suman Lata Joshi \\ Asst. Professor \\ Department of CSE/IT \\ SRMSCET, Bareilly
}

\author{
Chaitali Bhowmik \\ Asst. Professor \\ Department of CSE/IT \\ SRMSCET, Bareilly
}

\begin{abstract}
Token ring and token bus networks are two of the most commonly used type of local-area network (LAN). Token ring is designed in such a way that it can provide high throughput under heavy loads. Token bus is implemented using the token ring protocol over a virtual ring on a coaxial cable. The names, token ring and token bus, are applied to LANs which support a multiple access system using a token passing scheme. It is possible to define the maximum access delay experienced by a packet and thus to define the end-to-end delay across the network. In this paper after simulating the token ring and token bus technologies we have focused on the performance issues like throughput, mean delay, normalized throughput and response time of both the techniques. Lastly using some graphs and the data deduced from our simulation we have compared and concluded that token ring performs better than token bus for multi hop network design.
\end{abstract}

\section{Keywords}

Local Area Network; Mean Delay; Normalized Throughput; Response Time; Throughput; Token Bus; Token Ring

\section{INTRODUCTION}

A local-area network that is a geographically confined communication system can be characterized by the following components:

A transmission medium is being shared among different hops providing a broadcast capability; the Medium Access Control (MAC) protocol [1], that controls access to the medium and provides recovery mechanisms where needed; and a set of cooperating LAN interfaces through which hops attach to the network. It executes the MAC protocol and interface with the attaching hops. Access to the medium is monitored by a protocol called Carrier Sense Multiple Access with Collision Detection (CSMA/CD). Under a CSMA/CD protocol [7], every hop wanting to transmit a packet must listen to the channel to detect whether any transmission is in progress. In this case, it will defer its transmission until completion of the current transmission, otherwise it starts transmitting.

Despite carrier sensing, collisions cannot be completely avoided owing to the nonzero propagation delay of the bus.

Upon detection of a collision, transmission is aborted and the hop schedules its packet by determining a random retransmission interval, after which it makes another retransmission attempt. An alternative to bus system is LANs built on ring topology [7]. A ring network design consists of a collection of hops connected by unidirectional transmission channels to form a closed path. Information on the ring passes from hop to hop and is regenerated as they pass through each hop [2].

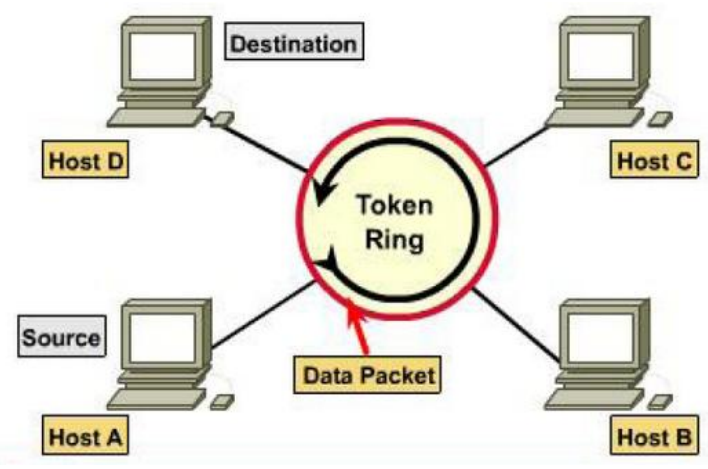

Figure 1. Token Ring design process [5]

The local network architecture considered here is illustrated in Fig. 2.

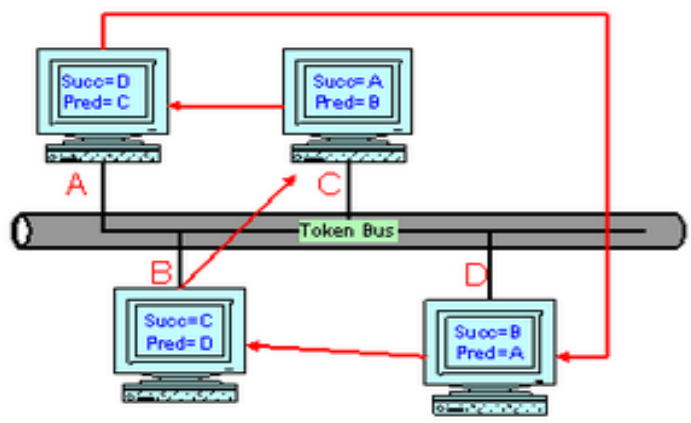

Figure 2. Token Bus design process [3]

It contains a set of four hops connected to a single channel. Each hop is assumed to be able to transmit through the channel only at a time. Hops are assumed to be equally distributed along the length of the bus [6] [8] so that the propagation delays between hops are equal. Also, the channels are assumed to be error-free and the network in steady state operation so that we do not consider acknowledgement traffic or topology maintenance.

The next section addresses the proposed token ring and token bus simulation model and describes the various components of ring and bus architecture. Section III demonstrates the performance analysis and comparison of token ring and token bus multi hop LAN technologies. Section IV concludes the paper.

\section{PROPOSED SIMULATION MODEL}

The functionalities of the simulator are based upon the Medium Access Control (MAC) layer [4] definition of the IEEE 802 standard [1]. We are interested in the throughput, Normalized throughput, transfer delay, response time of token 
bus and token ring network with four transmitting hops. The hop receives the necessary configuration information from the Hop Management Services including the slot-time [5] and other variables which are used in the maintenance of the ring and bus.

The token ring is being implemented using ring topology and the token bus is being implemented as a logical star topology. Interconnected token bus and token ring networks exhibit a number of characteristic performance properties.

The network design underlying our considerations is depicted in Fig. 1 and 2 as an example. In such a network, the routing problems are trivial, since only one path exists between any two hops. Hops are only attached to a main hub to form the desired topology. For simplicity we have used broadcasting rather than multicasting or unicasting as transmission type. We have assumed that no errors are being introduced and all the packets are being transmitted i.e. there are no unsent packets.

\section{COMPARISON AND \\ PERFORMANCE ANALYSIS}

To evaluate the performance of the token ring and token bus protocol, we consider performance measures such as the network throughput, mean delay, queuing time, transmission time, response time. However, due to lack of space, not all the results are reported. The basic network parameters considered here are the: topology, transmission type, payload information such as frame/frame time, frame/sec

For the following simulation results, the transmission type is set as broadcast. The data size / frame (in bytes) are considered as 18174 bytes. The frame/frame time is taken as 0.25 with $200 \mathrm{kbps}$ and $100 \mathrm{frame} / \mathrm{sec}$ as the payload. The results are presented in Table 1 .

Table 1. Comparison b/w parameters of token bus and token ring

\begin{tabular}{|l|l|l|}
\hline Parameters & Token bus & Token ring \\
\hline $\begin{array}{l}\text { Transmitting } \\
\text { hops }\end{array}$ & 4 & 4 \\
\hline $\begin{array}{l}\text { Frames } \\
\text { Generated }\end{array}$ & 3978 & 3978 \\
\hline
\end{tabular}

\begin{tabular}{|l|l|l|}
\hline Data Rate & 10 Mbps & $16 \mathrm{Mbps}$ \\
\hline Simulation Time & 29959202 & 36207795 \\
\hline $\begin{array}{l}\text { Normalized } \\
\text { Throughput (\%) }\end{array}$ & 86.82 & 99.83 \\
\hline Throughput (\%) & 87.02 & 99.97 \\
\hline $\begin{array}{l}\text { Mean delay } \\
\text { (Micro Sec) }\end{array}$ & 9885473.98 & 13046929.71 \\
\hline $\begin{array}{l}\text { Response time } \\
\text { (Micro Sec) }\end{array}$ & 29647.4 & 36380.51 \\
\hline Collision count & N/A & 0 \\
\hline Unsent frame & 0 & N/A \\
\hline
\end{tabular}

In fig 3 the network performance for token bus simulation is presented where we have considered 4 nodes only. The data rate is $10 \mathrm{Mbps}$. The Fig. 4 shows the performance for token ring where the data rate is fixed as $16 \mathrm{Mbps}$.

Fig. 5 to 8 shows the bar charts that are generated from the simulation. In all the graphs, we observe that the token ring is performs better in case of throughput. In multi hop token bus and token ring protocols, a significant portion of the transfer delay is due to the queuing time rather than transmission delay or propagation delay.

In Fig. 5, the transmitting nodes are plotted along the $\mathrm{X}$-axis and mean delay which is expressed in microsecond is plotted along Y-axis. Keeping the number of nodes fixed we can observe that token bus is having a mean delay of 9885473.98 Microsecond where token ring is having a mean delay of 13046929.71.

In Fig. 6, the transmitting nodes are plotted along the $\mathrm{X}$-axis and normalized throughput in percentage $(\%)$ is plotted along $\mathrm{Y}$-axis. From the bar chart it is observed that token bus is having a normalized throughput of $86.82 \%$ which is much less than the normalized throughput of token ring having $99.83 \%$ normalized throughput. 


Metrics Calculation
\begin{tabular}{|l|r|}
\hline Network Performance \\
\hline PayLoad Delivered (Bytes) & 32516172.000000 \\
\hline Overheads (Bytes) & 75582.000000 \\
\hline Queuing Time (MicroSec) & 39206478106.612831 \\
\hline Medium Access Time (MicroSec) & 91863964.592731 \\
\hline Transmission Time (MicroSec) & 26073403.199998 \\
\hline Dropped Frame(s) & 0 \\
\hline Error Frame(s) & \\
\hline Simulation Time (MicroSec) & 3978 \\
\hline Data Frames Generated & 86.827873 \\
\hline Normalized Throughput (\%) & 2965.029700 \\
\hline Throughput (\%) & 9865473.975466 \\
\hline Mean Delay (MicroSec) & \\
\hline Response Time (MicroSec) & \\
\hline \hline
\end{tabular}

Figure 3. Token Bus Simulation Result

Metrics Calculation
\begin{tabular}{|l|r|}
\hline Network Performance & 72296172.000000 \\
\hline PayLoad Delivered (Bytes) & 103428.000000 \\
\hline Qverheads (Bytes) & 51755964695.738686 \\
\hline Medium Access Time (MicroSec) & 108520636.733631 \\
\hline Transmission Time (MicroSec) & 36201043.125000 \\
\hline Dropped Frame(s) & 0 \\
\hline Error Frame(s) & 36207795.437500 \\
\hline Simulation Time (MicroSec) & 3978 \\
\hline Data Frames Generated & 99.835092 \\
\hline Normalized Throughput (\%) & 99.977918 \\
\hline Throughput (\%) & 13046929.707289 \\
\hline Mean Delay (MicroSec) & 36380.512785 \\
\hline Response Time (MicroSec) & \\
\hline \hline
\end{tabular}

Figure 4. Token Ring Simulation Result

Fig.7 and 8 show the plotting of transmitting nodes Vs response time and transmitting nodes $\mathrm{Vs}$ throughput respectively.

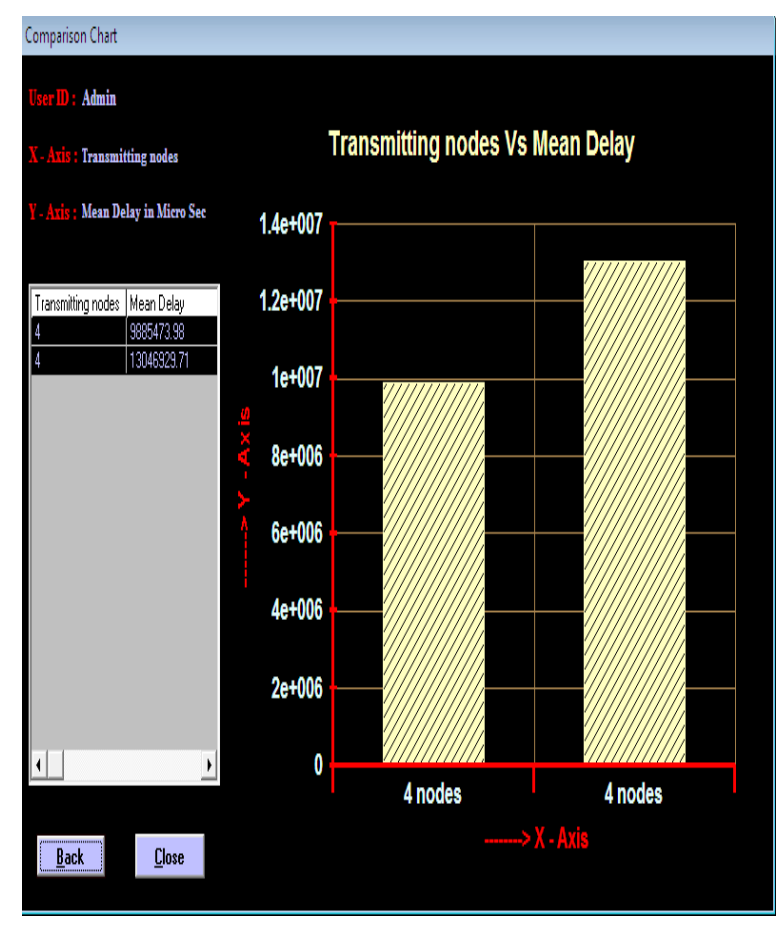

Figure 5. Transmitting node V/s Mean Delay comparison chart

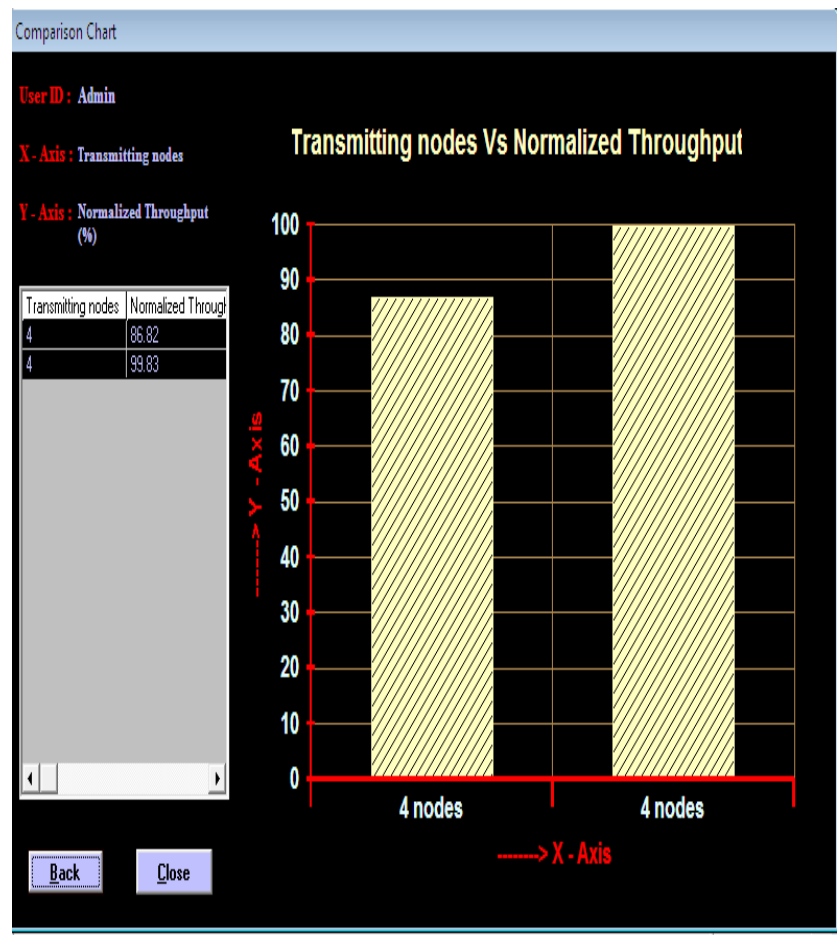

Figure 6 . Transmitting node V/s Normalized throughput comparison chart 


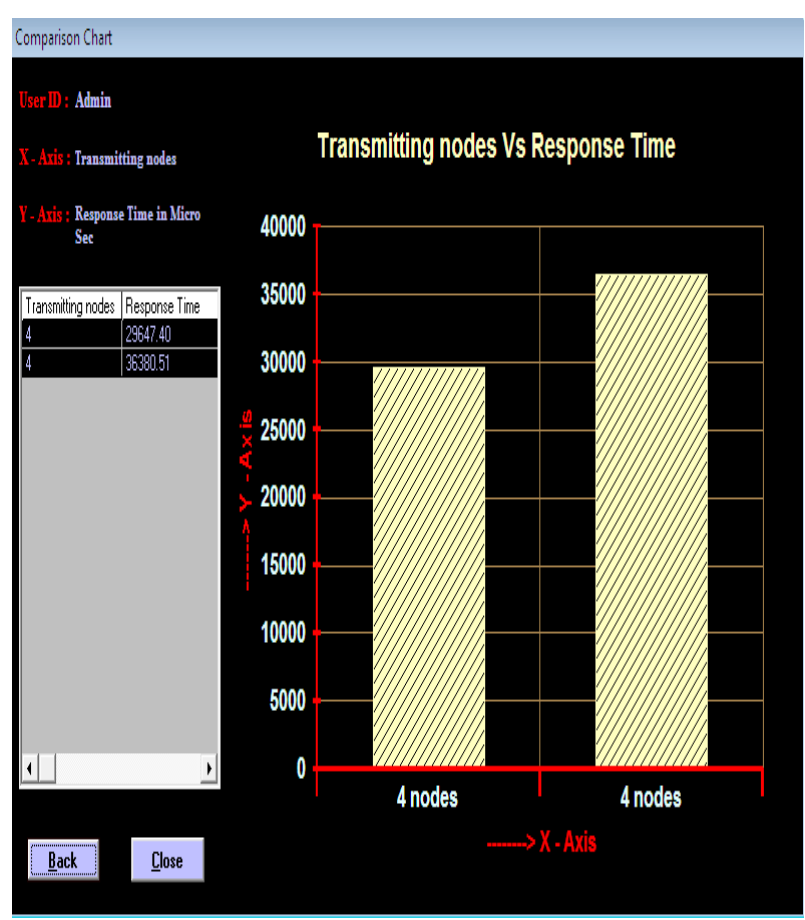

Figure 7. Transmitting node V/s Response time comparison chart

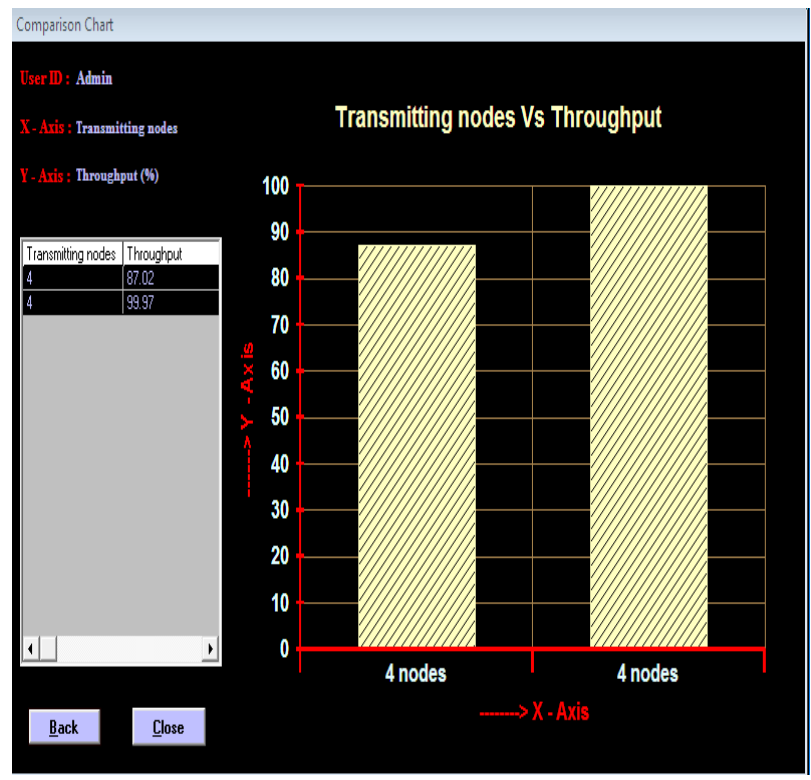

Figure 8. Transmitting node V/s throughput comparison chart

\section{CONCLUSION}

In this paper, we have presented the multi hop token ring and token bus LAN technologies. We have simulated and evaluated the performance of both the techniques. The performance of the protocol has been measured relative to variety of traffic loads and network parameters using simulation techniques. From this study, we have shown that for a given network configuration, the achievable throughput, delay depends on the type of protocol used and the different network parameters.

Since last few years, substantial research work has been done to study the various aspects of the performance of token ring and token bus based LANs. This work is of immense significance to establish the fact that token-ring is better than token bus as a major LAN technology. Today, we have achieved a satisfactory understanding of most practically relevant performance related issues. A better theoretical foundation of a number of problems is still missing. For example Analytic models of the basic token-ring and token bus operation are typically limited to Poisson arrivals of single packets and often require independence assumptions. Models yielding more detailed performance measures than just mean delays are of equal importance. An area where only very little theoretical understanding has so far been achieved is the modeling of LANs.

To verify our analysis, we designed a simulation model and generalized our study to handle mean delay and normalized throughput as performance parameters. Using the bar charts we have established the facts. In future we are planning to implement the analytical model related issues to enhance our current research work.

\section{REFERENCES}

[1]. B.W. Stuck, E.A. Arthurs. 1985. A Communication Network Wormance Analysis er. Prentice-Hall.

[2]. M.E. Ulug. 1984. Comparison of Token Holding Time strategies for a Token passing BI.IS. h9c. IEEE computer Networking pp: 37-44.

[3]. S. Ayandeh. 1989. Local Communication Networks for Industrial Automation. Canadian Journal of Electrical \& Computer Engineering.

[4]. J. Pang, F.A. Tobagi. 1988. Throughput Analysis of a Timer-Controlled Token-passing Protocol under Heavy Load. IEEE INFOCOM.

[5]. M. Ergen, D. Sengupta and P. Varaiya. 2004. WTRP wireless token ring protocol. IEEE Transactions on Vehicular Technology. pp: $1863-1881$.

[6]. D. A. Menasce and L. Leite. 1984. Performance of isolated and interconnected token bus local area networks. Proc., IFIP Cong. Perf. Eual. pp: 167-175.

[7]. D. Lin. 2005. A Study of Ring-based Networks for Group Mutual Exclusion and Wireless Mesh Networks. Master Writing Project, Department of Computer Science. San Jose State University, Dec 2005.

[8]. S.R. Sachs, K. Kan, and J.A. Silvestor. 1985. Token Bus Protocol Performance Analysis and Comparison with other LAN $\sim$ Protocols. IEEE Conf. Rec. GLOBECOM, pp: 48.1.1-48.1.7. 